\title{
The burden of diabetes mellitus in KwaZulu-Natal's public sector: A 5-year perspective
}

\author{
S Pillay, ${ }^{1} \mathrm{MB}$ ChB, FCP (SA), MMed; E Lutge, ${ }^{2} \mathrm{MB} \mathrm{ChB}, \mathrm{MPH}, \mathrm{MSc}, \mathrm{PhD}, \mathrm{FCPHM}(\mathrm{SA}) ;$ C Aldous, ${ }^{3} \mathrm{PhD}$ \\ ${ }^{1}$ Department of Internal Medicine, Edendale Hospital, Pietermaritzburg, South Africa \\ ${ }^{2}$ Department of Health, Pietermaritzburg, South Africa \\ ${ }^{3}$ School of Clinical Medicine, University of KwaZulu-Natal, Durban, South Africa
}

Corresponding author: S Pillay (drspillay@iafrica.com)

Background. Diabetes mellitus (DM), together with its devastating complications, has a huge impact on both the patients it affects and the global economy as a whole. The economies of developing countries are already under threat from communicable diseases. More needs to be done to stem the tide of non-communicable diseases like DM. In order for us to develop new strategies to tackle this dread disease we need to obtain and analyse as many data as possible from the geographical area where we work.

Objective. To describe the burden of DM in the public sector of the province of KwaZulu-Natal (KZN), South Africa (SA).

Method. Data on the number of diabetes visits, DM patients that were initiated on treatment, defaulters and DM-related amputations were accessed from the Department of Health records for the period 2010 - 2014 inclusive.

Results. There was a decline in the number of patients initiated on treatment per 100000 population from 2010 to 2014 inclusive (265.9 v. 197.5 v. 200.7 v. 133.4 v. 148.7). Defaulter rates for 2013 compared with 2014 were $3.31 \%$ v. $1.75 \%$, respectively and amputation rates were $0.09 \%$ v. $0.05 \%$ for 2013 and 2014, respectively. There was a strong proportional relationship observed between the number of defaulters and number of diabetes-related amputations $(r=0.801 ; p=0.000)$ (Pearson correlation). A notable percentage of DM patients ranging between $63 \%$ and $80 \%$ were commenced on pharmacological therapy at their local clinics rather than at hospitals in the province.

Conclusion. Strategies directed towards detection and treatment of DM, together with decreasing defaulter rates and thereby decreasing diabetes-related amputations, need to be addressed urgently. The majority of patients were initiated on therapy at the clinic level. This emphasises the need to strengthen our clinics in terms of resources, staffing, and nursing and clinician education, as this is where diabetes control begins. Although this study was based solely in $\mathrm{KZN}$, the second most populous province in SA, it probably reflects the current situation regarding DM in other provinces of SA as well.

S Afr Med J 2016;106(4):384-388. DOI:10.7196/SAMJ.2016.v106i4.9920

Diabetes mellitus (DM) is a chronic non-communicable disease (NCD) that has serious long-term complications and results in significant economic costs for patients and health systems alike worldwide. ${ }^{[1]}$ Developing countries will do well to prevent and control this disease to limit its impact on health systems already under immense burden from communicable diseases like tuberculosis and HIV infection.

The International Diabetes Federation (IDF) estimated that there were 22 million diabetes patients living in Africa in 2014, excluding the $62 \%$ of patients that are undiagnosed. ${ }^{[2]}$ In South Africa (SA) DM accounts for 58 deaths daily and is the fifth highest cause of natural deaths. ${ }^{[3]}$

DM is increasing at an alarming rate worldwide, especially in developing countries, where among other reasons increased urbanisation and westernised diets play an important role in the aetiology of this disease. ${ }^{[4]}$ Poor diabetic control will increase the risk of diabetes-related complications like retinopathy and peripheral arterial disease, requiring laser surgery and amputations, respectively. ${ }^{[5]}$ One of the reasons for poor diabetic control is patients defaulting regular treatment at clinics and hospitals.

The vast majority of diabetes patients are still not achieving adequate glycaemic, lipid and blood pressure control at both public and private institutions. ${ }^{[6,7]}$ Following the Brazzaville declaration on NCD prevention, governments worldwide have committed to a more vigorous and sustained approach to NCDs; this is similar to the approach that was used in the antiretroviral roll-out for HIV infection in SA. ${ }^{[8]}$
Analyses of data relating to new cases of DM and some of the complications encountered in KwaZulu-Natal (KZN) over the last 5 years would be beneficial in helping healthcare professionals and government assess the burden of DM in this province.

Diabetes data are routinely collected from all public health facilities in KZN, as part of the District Health Information System (DHIS). These data are aggregated at facility level and no individual patient data are used. The data are managed by the Data Management Unit of the KZN Department of Health. Data from the DHIS are available from the Data Management Unit on request.

In this retrospective study we accessed records from the DHIS over the period of 5 years (2010 - 2014, inclusive), confirming the number of new diabetes cases and the total number of patients seen in the relevant health institutions. In addition, for the last 2 years of the study period, the number of defaulters and diabeticrelated amputations performed for the entire province of KZN were analysed.

\section{Methods \\ Study setting}

This was a province-wide study looking at data from facilities which contribute data to the DHIS. KZN is one of the nine official provinces of SA and has a population of approximately 10.5 million people; it is the second most populous province in SA. ${ }^{[9]}$ The province has been divided into one metropolitan municipality (eThekwini) and 10 district municipalities. 
Each local municipality (LM) has its own healthcare facilities which could include one or more of the following:

- Clinics

- District hospital

- Gateway clinic

- Regional hospital

- Specialised chronic hospital

- Specialised tuberculosis (TB) hospital

- Community health centre

- Provincial tertiary hospital

- Specialised psychiatric hospital.

\section{Data collection}

Ethics approval for the study was obtained from the local ethics review committee (Biomedical Research and Ethics CommitteeBREC) and Department of Health. Data were then accessed from DHIS records.

For the purposes of this study, the following indicators were analysed for each district of KZN:

- Number of new patients initiated on treatment

- Number and age of new patients initiated on treatment at clinics v. hospitals

- Number of laser surgeries performed at hospitals

- Number and percentage of diabetes-associated amputations

- Number and percentage of defaulters.

These data were interrogated to assess the current burden of DM and diabetes-associated amputations in KZN using simple descriptive statistics.

\section{Results}

The total number of new patients initiated on treatment per year, combined for all municipalities of KZN, is shown in Fig. 1. Fig. 2 demonstrates the number of new patients initiated on treatment per 100000 population. Fig. 3 and Table 1 illustrate the number of new diabetic patients initiated on treatment in each of the municipalities of KZN. Table 2 illustrates the current pattern of initiation of treatment of diabetes in SA; the majority of patients are diagnosed and treatment is initiated at clinic level.

From 2012, data collection increased to include the number of laser surgeries performed at the various healthcare facilities. There were 1584 diabetes-related laser surgeries performed at hospitals within the province of KZN during 2012.

From 2013 onwards, data collection from the various healthcare centres was expanded to include number of amputations, number of defaulters and ages of patients started on medication. The majority of patients started on medication in 2013 and 2014 were over 18 years of age (90.4\% and $92.6 \%$, respectively). Defaulter and amputation rates for 2013 and 2014 are listed in Table 3.

\section{Discussion}

Currently 387 million patients suffer from DM globally; the majority live in low- and middle-income countries. ${ }^{[2]}$ Combined unemployment rates in the metropolitan municipality and 10 district municipalities of KZN were far greater than the overall unemployment rates for SA reported by Statistics SA (32.5\% v. $24.3 \%) .{ }^{[10]}$ Poverty can lead to lack of access to healthcare and directly impact on diabetes control and complications. DM, with its complications, is both a serious and costly NCD worldwide. Understanding the magnitude and severity of this disease requires access to as many data as possible in Africa, where up to $62 \%$ of diabetes remains undiagnosed. ${ }^{[2]}$ Analysis of our data allowed assessment of what the current state of

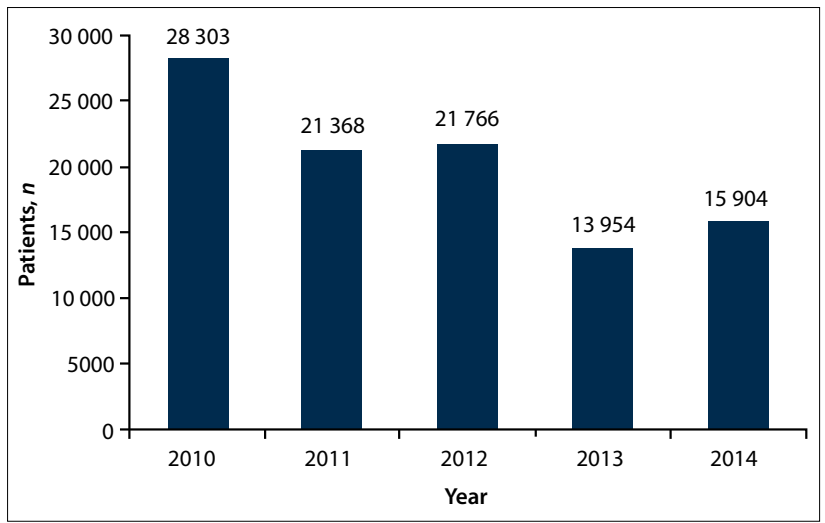

Fig. 1. New patients initiated on treatment per year, combined for all municipalities of KZN.

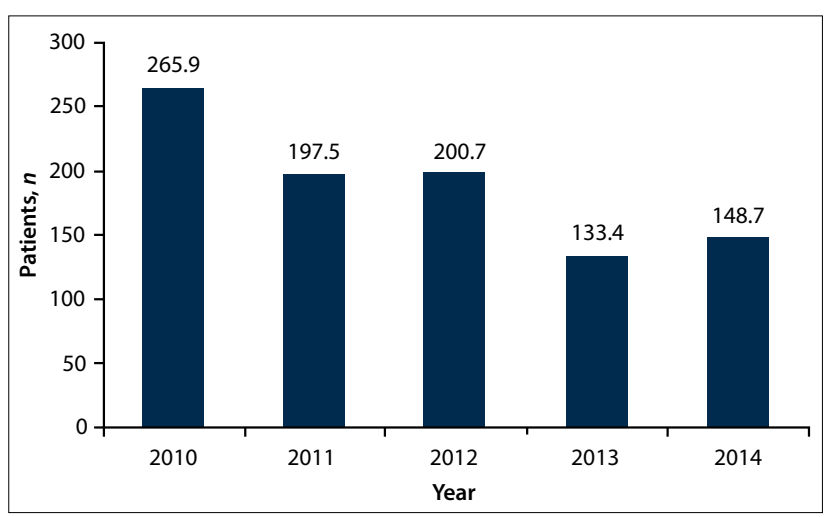

Fig. 2. Number of new patients initiated on treatment per 100000 population.

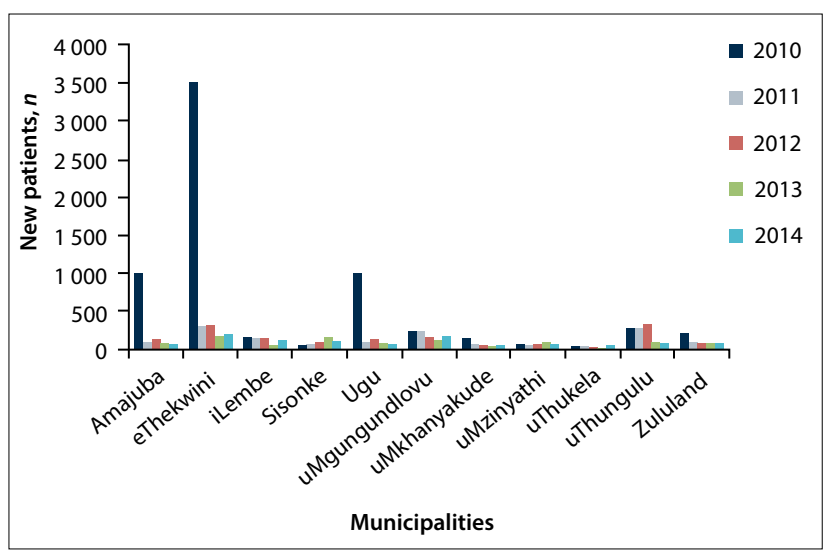

Fig. 3. New diabetic patients initiated on treatment in each of the municipalities of $K Z N$.

$\mathrm{DM}$ is in $\mathrm{KZN}$ in order to develop improvement strategies to combat this deadly disease. This study included data from all state sector healthcare facilities in KZN.

Our study demonstrates that the majority of diabetes patients (ranging between 63\% and 80\%) are diagnosed at local clinics, and initial management of their diabetes was instituted at this level. These clinics are often resource-limited and nurse-orientated. Intervention strategies to control DM need to be intensified at this level because very often by the time the patient visits a higher level of healthcare they have developed long-term complications from DM. Bearing in mind that these clinics are resource-limited, emphasis needs to be placed on both nurse and patient education, together with simple approaches such as introducing standardised clerking sheets with 
Table 1. New cases of diabetes initiated on treatment per 100000 population per district from 2010 to 2014, $n$

\begin{tabular}{llllll}
\hline & $\mathbf{2 0 1 0}$ & $\mathbf{2 0 1 1}$ & $\mathbf{2 0 1 2}$ & $\mathbf{2 0 1 3}$ & $\mathbf{2 0 1 4}$ \\
\hline Amajuba & 1013.0 & 110.8 & 139.7 & 87.0 & 66.3 \\
eThekwini & 3516.0 & 315.9 & 322.8 & 179.3 & 207.7 \\
iLembe & 172.0 & 150.4 & 152.2 & 66.0 & 127.9 \\
Sisonke (Harry Gwala) & 63.1 & 85.1 & 105.7 & 168.0 & 113.7 \\
Ugu & 154.2 & 138.2 & 140.4 & 142.7 & 183.9 \\
uMgungundlovu & 251.7 & 254.7 & 167.4 & 114.4 & 182.4 \\
uMkhanyakude & 149.2 & 78.4 & 62.5 & 54.6 & 62.9 \\
uMzinyathi & 77.5 & 61.7 & 65.3 & 94.8 & 66.5 \\
uThukela & 44.2 & 43.1 & 42.3 & 33.4 & 56.9 \\
uThungulu & 273.1 & 274.3 & 331.5 & 106.3 & 81.8 \\
Zululand & 228.8 & 95.6 & 90.6 & & 83.2
\end{tabular}

Table 2. Patients started on treatment in clinics $(\mathrm{C}) \mathrm{v}$. hospitals $(\mathrm{H})$ over the study period in the various districts of KZN, $n$

\begin{tabular}{|c|c|c|c|c|c|c|c|c|c|c|}
\hline \multirow[b]{2}{*}{ Districts of KZN } & \multicolumn{2}{|c|}{2010} & \multicolumn{2}{|c|}{2011} & \multicolumn{2}{|c|}{2012} & \multicolumn{2}{|c|}{2013} & \multicolumn{2}{|c|}{2014} \\
\hline & $\mathrm{C}$ & $\mathbf{H}$ & $\mathrm{C}$ & $\mathbf{H}$ & C & $\mathbf{H}$ & C & $\mathbf{H}$ & C & $\mathbf{H}$ \\
\hline Amajuba & 445 & 4125 & 509 & 0 & 653 & 0 & 414 & 0 & 321 & 0 \\
\hline eThekwini & 10961 & 1620 & 8863 & 2114 & 7316 & 4517 & 5347 & 1345 & 5905 & 1986 \\
\hline iLembe & 511 & 416 & 689 & 136 & 668 & 182 & 288 & 87 & 480 & 260 \\
\hline Sisonke (Harry Gwala) & 199 & 123 & 442 & 0 & 368 & 191 & 224 & 680 & 318 & 305 \\
\hline Ugu & 1007 & 110 & 755 & 264 & 721 & 333 & 798 & 292 & 767 & 663 \\
\hline uMgungundlovu & 1886 & 653 & 2615 & 0 & 1644 & 106 & 1537 & 0 & 1825 & 151 \\
\hline uMkhanyakude & 686 & 249 & 494 & 6 & 300 & 106 & 299 & 62 & 294 & 129 \\
\hline uMzinyathi & 327 & 65 & 318 & 0 & 342 & 0 & 181 & 325 & 262 & 99 \\
\hline uThukela & 322 & 0 & 320 & 0 & 320 & 0 & 210 & 47 & 324 & 122 \\
\hline uThungulu & 1052 & 1439 & 1482 & 1065 & 1047 & 2087 & 729 & 294 & 659 & 156 \\
\hline Zululand & 701 & 1406 & 674 & 222 & 699 & 166 & 301 & 494 & 651 & 227 \\
\hline Total (\%) & $\begin{array}{l}18097 \\
(63.94)\end{array}$ & $\begin{array}{l}10206 \\
(36.06)\end{array}$ & $\begin{array}{l}17161 \\
(80.31)\end{array}$ & $\begin{array}{l}4207 \\
(19.69)\end{array}$ & $\begin{array}{l}14078 \\
(64.68)\end{array}$ & $\begin{array}{l}7688 \\
(35.32)\end{array}$ & $\begin{array}{l}10328 \\
(74.01)\end{array}$ & $\begin{array}{l}3626 \\
(25.99)\end{array}$ & $\begin{array}{l}11806 \\
(74.23)\end{array}$ & $\begin{array}{l}4098 \\
(25.77)\end{array}$ \\
\hline
\end{tabular}

Table 3. Defaulter and diabetes amputation rates for 2013 and 2014

\begin{tabular}{|c|c|c|c|c|c|c|}
\hline \multirow[b]{2}{*}{ Districts of KZN } & \multicolumn{3}{|c|}{2013} & \multicolumn{3}{|c|}{2014} \\
\hline & $\begin{array}{l}\text { DM patients } \\
\text { on register }(N)\end{array}$ & Defaulters, $n(\%)$ & $\begin{array}{l}\text { Diabetes-related } \\
\text { amputations, } n(\%)\end{array}$ & $\begin{array}{l}\text { DM patients } \\
\text { on register }(N)\end{array}$ & Defaulters, $n(\%)$ & $\begin{array}{l}\text { Diabetes-related } \\
\text { amputations, } n(\%)\end{array}$ \\
\hline Amajuba & 51741 & $2285(4.42)$ & $58(0.11)$ & 57952 & $4883(8.43)$ & $42(0.07)$ \\
\hline eThekwini & 480531 & $10259(2.13)$ & $527(0.11)$ & 518878 & $9714(1.87)$ & $464(0.09)$ \\
\hline iLembe & 83783 & $6312(7.53)$ & $76(0.09)$ & 87144 & $2937(3.37)$ & $109(0.13)$ \\
\hline Sisonke (Harry Gwala) & 41739 & $2034(4.87)$ & $4(0.01)$ & 48220 & $2017(4.18)$ & $2(0.004)$ \\
\hline Ugu & 103331 & $7003(6.78)$ & $43(0.04)$ & 111790 & $3868(3.46)$ & $105(0.09)$ \\
\hline uMgungundlovu & 152345 & $4402(2.89)$ & $125(0.08)$ & 152263 & $8551(5.62)$ & $254(0.17)$ \\
\hline uMkhanyakude & 36919 & $2458(6.66)$ & $29(0.08)$ & 40364 & $2452(6.07)$ & $21(0.05)$ \\
\hline uMzinyathi & 44917 & $898(2.00)$ & $7(0.02)$ & 50809 & $992(1.95)$ & $8(0.02)$ \\
\hline uThukela & 52852 & $1976(3.74)$ & $49(0.09)$ & 48368 & $2429(5.02)$ & $16(0.03)$ \\
\hline uThungulu & 137264 & $2253(1.64)$ & $165(0.12)$ & 149472 & $1435(0.96)$ & $167(0.11)$ \\
\hline Zululand & 67246 & $1537(2.29)$ & $5(0.01)$ & 56910 & $957(1.68)$ & $10(0.02)$ \\
\hline Total & 1252668 & $41417(3.31)$ & $1088(0.09)$ & 2298417 & $40235(1.75)$ & $1198(0.05)$ \\
\hline
\end{tabular}


clear referral criteria highlighted so that all aspects of diabetes management are standardised. These clinics should receive increased staffing in the form of doctor support and nursing, which is the way forward envisaged by the National Health Insurance (NHI). Since the majority of patients are being initiated on diabetes management at clinic level, the above steps will ensure that the appropriate management begins at this level of primary care.

Our study revealed that there is still a high prevalence of defaulters in 2013 and 2014 (3.3\% and $1.8 \%$, respectively). These defaulter rates correlate well with defaulter rates seen at clinics in the UK, where they range between $3.5 \%$ and $4.1 \% \cdot{ }^{[1]}$ When the severity of this disease, together with its complications and the burden it places on the economy, is taken into account, this high number of patients defaulting needs to be urgently addressed. Possible reasons for high defaulter rate may include:

- Poor patient education on the disease and its potentially lifethreatening complications.

- Long distances for patients to travel to healthcare facilities (Zgibor et al. ${ }^{[12]}$ in their study found that the greater the distance a rural patient had to travel to healthcare facilities, the poorer the glycaemic control that was achieved).

- Poor socio-economic status of patients in this province with high rates of unemployment noted in the last census.

- Patients with concomitant communicable diseases such as TB and HIV may experience difficulty in attending diabetic clinics due to physical inability or they might find it difficult to attend multiple healthcare institutions on a monthly basis for each medical condition. Hence the urgent need for combined communicable and non-communicable disease clinics in SA.

Our study further showed that the prevalence of defaulters actually decreased between 2013 and 2014 (3.3\% v. 1.8\%, respectively). Whether this trend will continue in future years is still to be seen as only 2 years of defaulter data were available. As expected with the defaulter rate decreasing from 2013 to 2014, amputation rates also decreased accordingly $(0.09 \%$ v. $0.05 \%$, respectively). There is a strong proportional relationship between number of defaulters and number of diabetes-related amputations $(r=0.801 ; p=0.000)$ (Pearson correlation).

The number of patients initiated onto treatment declined notably between 2010 and 2014, contradicting the global incidence of diabetes which is on an upward spiral. ${ }^{[2]} \mathrm{A}$ few possible explanations for this phenomenon might be that the health system is not detecting new patients or that there was an error during data collection and it does not reflect the true number of new patients. The unemployment rates for KZN are much higher than the overall rates for the country $(32.5 \%$ v. $24.3 \%$, respectively) and hence poverty and the lack of access to healthcare might also help explain why the number of new patients starting on treatment is decreasing within the province. According to the latest IDF figures, approximately $62 \%$ of diabetic patients in Africa are undiagnosed ${ }^{[2]}$ and the downward trend noted in detecting new cases in KZN is a warning sign that improved systems need to be implemented in order to increase early detection and treatment of diabetes in the community and the province as a whole. More emphasis needs to be placed on prevention of this disease (lifestyle modification through diet and exercise). With increasing urbanisation and adoption of Western diets, many of the patients of African descent are now prone to chronic lifestyle diseases such as DM. ${ }^{[4,13]}$

The decreasing trend noted in the number of new diabetic patients initiated on treatment in Figs 1 and 2 was largely attributed to a considerable decrease in the number of new diabetic patients initiated on treatment in two main municipalities (eThekwini and Amajuba) illustrated by Fig. 3 and Table 1. This trend could be explained by failure to identify new diabetic patients by our healthcare systems.

Most of the diabetes-related laser surgeries (93.1\%) were performed within the larger eThekwini metropolitan municipality. This is possibly an indication that the number of laser surgeries performed needs to be increased at other regional hospitals within the province, thereby alleviating the burden on the metropolitan hospitals and making this sight-saving surgery available to the greater population at large.

Van Houtum et al. ${ }^{[14]}$ found that diabetic patients are 20 times more prone to lower-limb amputations (LLA) than non-diabetics. $\mathrm{DM}$ is regarded as the most common aetiology of non-traumatic LLA worldwide and in SA DM is thought to be responsible for around $60 \%$ of LLA. ${ }^{[15]}$ Factors such as poverty, poor sanitation and walking barefoot may be responsible for increased amputation rates in developing countries. ${ }^{[16]} \mathrm{KZN}$ has a high unemployment rate; it is possible that a large number of patients are walking barefoot, which increased their risks of LLA. Statistics SA found that approximately half (46.8\%) of the population of KZN had no access to flush toilets connected to sewerage. ${ }^{[2]}$ When the abovementioned social factors of poverty, walking barefoot and poor sanitation, and the overall poor control of both blood sugar and blood pressure found in our clinics are taken into account, ${ }^{[6-7]}$ it is not surprising to note that there is increasing evidence of LLA. This trend is expected to increase if measures are not implemented to achieve better control of both glycaemia and BP and improvement of socio-economic factors.

Amputations affect patients' physical and emotional status and increase premature mortality. ${ }^{[17]}$ They place a huge burden on the economy of a country. As a result of amputations patients often lose their employment, resulting in a loss of income for the family. Economic costs of an amputation range from in-hospital stay, which may or may not include intensive care stay, to the rehabilitation of the patient and cost of prosthesis. Complications from the surgery may also increase the duration of hospital stay, further increasing the cost to the state. Prevention and treatment of foot complications early in the course of disease are paramount in avoiding amputations. Patient education on foot care is an essential part of diabetes care and begins at community-clinic level. Not only do we need education for patients but more training for nursing staff and clinicians who serve as first contact for all diabetic patients. As was seen in this study, amputation rates decrease with a decline in defaulter rates, which is a powerful indicator that strategies to decrease defaulter rate will help minimise the burden of DM both on the patient and the economy.

\section{Conclusion}

Good control of DM requires that patients attend clinics regularly, make lifestyle changes and be pro-active in their treatment programme. The high unemployment rate in KZN, coupled with the HIV and TB disease burden, makes it almost impossible to achieve treatment goals and prevent complications. Good control begins in the local healthcare clinics which serve as first point of contact for the majority of the population. First contact with these clinics and their staff often determines whether a patient stays in the programme or defaults. We need to strengthen this first point of contact and make sure that patient education is maximised at this level, together with improving clinical staff compliance with local diabetes guidelines. Specific criteria need to be used to help up-referrals to district, regional and tertiary healthcare centres. Methods to decrease defaulter rates need to be implemented at all levels of healthcare in order to decrease complications like LLA and retinopathy. 


\section{References}

1. Jonsson B. The economic impact of diabetes. Diabetes Care 1998;21(3):C7-C10. DOI:10.2337/ diacare.21.3.C7

International Diabetes Federation. Diabetes Atlas. 2014. http://www.idf.org/diabetes atlas (accessed 1 May 2015)

3. Statistics South Africa. South African Statistics 2012. http://www.statssa.gov.za/publications/ SAStatistics/SAStatistics2012.pdf (accessed I May 2015).

4. Steyn K, Kazellenbogen JM, Lombard CJ, Bourne LT. Urbanization and the risk for chronic diseases of lifestyle in the black population of the Cape Peninsula, South Africa. Eur J Preventive Cardiol 1997;4(2):135-142. DOI:10.1177/174182679700400210

5. American Diabetes Association. Implications of United Kingdom prospective diabetes study. Diabetes Care 2002;25(Suppl 1):S28-S32. http://care.diabetesjournals.org/content/25/supp_1/s28. full (accessed 1 May 2015)

6. Pillay S, Aldous C, Mahomed F. Diabetic patients served at a regional level hospital: What is their clinical picture? J Endocrinol Metab Diabetes S Afr 2015;20(1):60-66.

7. Amod A, Riback W. Diabetes guidelines and clinical practice: Is there a gap? The South African cohort of the International Diabetes Management Practices Study. J Endocrinol Metab Diabetes S Afr 2012;17(2):85-90.

8. World Health Organization. Brazzaville Declaration on Noncommunicable Diseases. 2011. www.afro. who.int/en/media-centre/pressreleases/2839-african-health-ministers-adopt-brazzaville-declarationon-noncommunicable-diseases.htm (accessed 1 May 2015).
9. Statistics South Africa. Mid-year population estimates, 2014. http://beta2.statssa.gov.za/publications/ P0302/P03022014.pdf. 31 July 2014.(accessed 1 May 2015).

10. Statistics South Africa. Unemployment estimates, 2015. http://www.statssa.gov.za/ (accessed 1 May 2015).

11. Hammersley MS, Holland MR, Walford S, Thorn PA. What happens to defaulters from a diabetic clinic? BMJ (Clin Res Ed) 1985;291(6505):1330-1332

2. Zgibor JC, Gieraltowski LB, Talbott EO, et al. The association between driving distance and glycemic control in rural areas. J Diabetes Sci Technol 2011;5(3):494-500. DOI:10.1177/193229681100500304 3. Bourne LT, Lambert EV, Steyn K. Where does the black population of South Africa stand on the nutrition transition? Public Health Nutr 2002;5(1A):157-162. DOI:10.1079/PHN2001288

4. Van Houtum WH, Lavery LA, Harkless LB. The impact of diabetes-related lower-extremity amputation in the Netherlands. J Diabetes Complications 1996;10(6):325-330. DOI:10.1016/1056-8727(95)00088-7

15. Islave A, Levitt N. Diabetes and lower extremity amputations in the Cape Town metropole public sector, South Africa. Diabetic Med 2006;24(4):411-607.

16. Boulton A. The diabetic foot: Epidemiology, risk factors and status of care. Diabetes Voice 2005;50:5-7.

17. Peters EJG Childs MR, Wunderlich RP, et al. Functionl stus of persons with dibetes - 2005 ded lower-

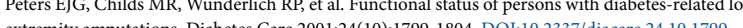

Accepted 22 October 2015. 